\title{
Inside Clinical Arena Automated Evaluation Using Selenium testing framework
}

\author{
Mr. Vinay Raj A.S \\ Assistant professor, Department of Computer science, MVJ College of Engineering. \\ Email: vinayrajas10@gmail.com
}

\begin{abstract}
Test automation is frequently used in software development process, as well as in other domains, specifically the medical sector. Various application programs were already designed for internet programmers that can be accessed via an internet browser. It has become progressively critical to control and enhance the quality of web applications. Automation testing minimizes testing costs while improving work productivity, leading to high, reliable products or services. Web applications enhances the program's sophistication, rendering manual testing impractical. This should considerably more. It's indeed difficult to get consistent results. It is possible to prevent this by utilizing automated testing. The aim of this article is to interpret test automation in development tools for hospital of administrators that used the selenium test automation tool. This is a compilation of testing tools for various browsers, software platforms and programming languages. Selenium is a test automation tool that may be used to create test cases for internet apps. It has the entirety of the features required to automated tests.
\end{abstract}

Keywords: Software testing, Web applications, Automation testing, Hospital management, Selenium

\section{INTRODUCTION}

Manual Testing is entirely reliant on manual representative. It subordinates on Analyst and Engineers to be associated in systemic series of tasks from the onset of the tasks or events to concrete final assassinate. Automated testing eliminates the essential for accustomed manual assassinate. Aside from software tools, the tasks can be put through using a test automation substructure. The primary variance allying both the testing mechanisms is the optimal time and the mode in which the tasks are carried out. Manual testing requires probably longer time than automated testing. In manual testing, the process is assassinated by an analyst and QA engineers. . However, with automated testing, analysts and QA engineers must install the software programme preceding to assassinate. As a result, the software does the execution. Manual testing is ineffectual in disparity to automated testing. It is arduous to ensure requisite test coverage. It is unadorned to achieve pertinent test analysis with automated testing. Face ID, location mimicking, fingerprint testing, and network virtualization are all examples of real-world user simulation [15]. Automated testing has numerous ascenders which comprise of firmly feedback cycle, less time consumption[4]. No substantial expenses in Business, greater test broadcasting, renewable of the deducted process, high accuracy, fast determination of stability of the build(smoke testing),elimination of manual error, etc., 


\section{AUTOMATION TESTING}

\subsection{SELENIUM FRAMEWORK}

The Selenium Platform is indeed an open source and freely automated test system for test automation. Selenium is indeed a set of software technologies which provides a different method to examine automating [5]. It enables users to manage along a variety of operating systems and browser.

\subsubsection{PROGRAMMING LANGUAGES}

It supports a wide range of programming languages, except ASP language as the element of the language does not trigger the function of the script page.

Though many languages still available and new languages are being created, we must note that learning $90 \%$ of the concepts in a specific language is also applicable to entire different language. When the core basics are familiar (Program design, operation of programming language, data structure and control structure).

\section{i. Python}

When comparing to the other programming languages, python is easy and having far less verbose. The APIs of python empowers the users to connect with the browser through selenium. Despite having variations in their browsers design selenium uses the standard commands in python to different browsers.

\section{ii. JAVA Script}

The test automation can be done because of wider adoption of shift-left methodologies, where developer can also be involved in test-code development. One of the major reasons for dominance JAVA script in test automation was shift-left testing. The QA teams work in collaboration with the developer team to get best efficient test automation in this shift-left method. End to end testing is the major thing makes java script a protected programming language for the test automation

\section{iii. C Programming}

This programming language is slow and steadily getting attention in the field of test automation. Many developers are considering $\mathrm{C}$ programming for the development of test cases to cross between browsers because of the availability of wide range of frameworks.

\section{iv. Ruby}

Like python ruby is also easy to learn, human-friendly, flexible, syntax and object-oriented architecture. This makes it a powerful programming language. It can be used to build a useful application with much lesser lines of codes. The execution of first case browser test with selenium web driver and ruby are done with very lines of codes 


\section{v. PHP}

When compared to other programming languages the difficulty level in PHP is very low. It has good growing system and community support. It gives XDebug, which is a powerful debugging and profiling tool that has powerful capabilities.

\section{vi. TestNG}

It generates the report in a proper format including number of tests, cases passed, cases failed, and skipped. Multiple tasks can be grouped and saved in .xml file type by which we can make priorities that which task have to done first. By using a key word called invocation count, a same task can be done multiple times without loops. This really is easy to integrate with programs including TestNG, Maven, and Jenkin.

\section{Groovy}

It is a dynamic and agile language. It integrated with all java objects and programs existed. The language is meaningful and concise when compared to JAVA.

\section{vii. JAVA}

It is a platform independent language because Java makes use of JVM. It can be operated in any system which has the JVM in it.

\subsubsection{COMPONENTS}

Selenium is made up of a number of components such as IDE, RC, Web driver and Grid. The Selenium IDE (Development Environment) is a Selenium legal trial development platform that also functions as an integration platform.. Selenium IDE is a Firefox insert that enables users to $\log$ actual activities while they go thru a procedure. Selenium RC is a UI which accepts Selenium commands from of the editors and performs testing inside the web [17]. More sophisticated testing requires the use of a range of languages. Selenium Web Driver is the follow-up to Selenium RC, and it sends instructions to a web server and receives responses. Selenium Grid is a solution that allows you to run tests in parallel on numerous PCs and browsers at the same time, resulting in a shorter execution time.

Web-based software, Comparing to desktop systems, need additional modifications, including such firmware upgrades, security problems, and preferences [13]. Testing phase for a entire systems is prohibitively expensive for corporations so because anticipated turnaround time for changes is low. Selenium is a web automation platform that is often used it to perform end-to-end web app testing. Website toolkits accomplish the job at hand and automate browser management, enabling again for automating of repetitive tasks [9]. This has an amount of characteristics that really are beneficial to programmers. Selenium IDE is used for recording, and for newbie's to a development industry. Selenium RC or Web Driver can be used by developers who are proficient in programming languages. The Parallel selenium tests can be done with selenium grids. By selecting the appropriate framework, one can save time and money while also improving software quality. In a software test plan, it is impossible to automate every test case. The testers must first identify which testing must be automated? As a consequence, tester first must determine what tests would be mechanized 
prior continuing. All GUI components, database connection, validations, as well as other tasks can be mechanized inside a cost-effective way. [14]. when selecting whether or not to automate testing, the following variables are taken into account: Products that necessitate repeating the same tests over and over. Product specifications do not change regularly. Programming languages can be used to automate processes. A number of tools are available to assist with test automation. For unit testing, the JUnit Automation Framework is commonly used. Selenium and Selenium Web Driver are used to test web applications. These tools aid in the development of a testing framework.

\subsection{IMPLEMENTATION OF TEST CASES}

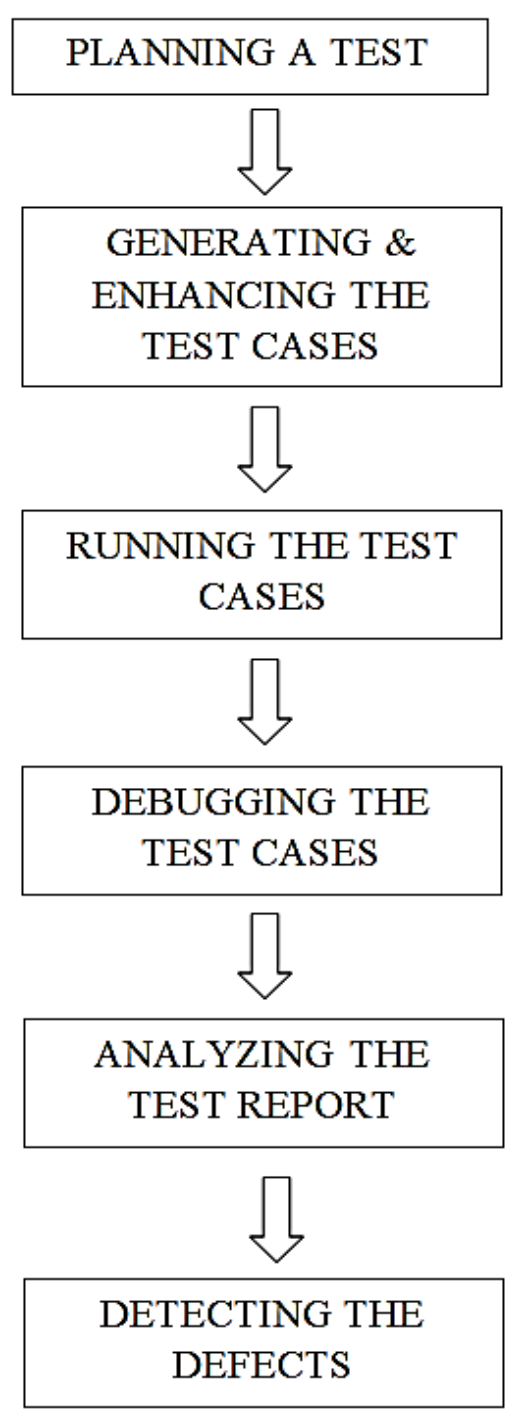

Fig.1: Techniques concerned in test case

\section{A. Inserting Verifications and Asserts to Your Documentation (using the Context Menu)} The experiments will involve examining the page's attributes. This necessitates the use of state and check status. The procedure for adding the necessitates in the test case is that, Correct everywhere on the site with Selenium-IDE record and go to an application which shows the test application. There may only be one Selenium charge reported the first time you use Selenium. 
Visit a web site of our choice and tap on the text box. Right-tap the material you want to use right now. The recommended parameter should be the content itself, and the connection menu should provide you should receive a complete content Program. The Available Commands menu option displays a slew of new orders, along with suggested parameters, for testing your currently selected UI component.

Experiment with a few more VI components. Access the client control, such as a catch or a checkbox to proceed forward. To display options other than verify Text present, you may need to use 'Show All Available Commands'. On choosing one of these options, Just on vital link option, the most frequently utilized connections would show.

Such purchases would be discussed in further detail detail inside the chapter on Selenium pricing. Again for moment term, record and pick requests into such a test in the IDE that you can then run. By looking at various aspects of an IDE, one can learn a lot about Selenium summons.

\section{B. Creating a Test Case}

Under the File menu, there are Save and Open commands. Selenium, on the other hand, recognizes experiments and test suites. We can either save and save Selenium-IDE experiments for future usage, users could save single tests or the entire test suite. If indeed the testing suite's trials haven't really been spared already, we'll be asked to do that before the testing procedure is saved[11].

Whenever users start a present experiment or suite, Selenium-IDE displays its Selenium charge inside the Test Case Pane.

\section{Test Cases in Execution}

The IDE provides a variety of options for performing your experiment. We can run multiple Experiments could be run in parallel, paused and resumed, ran single line by line, a solitary summon could be performed at a moment, and a test suite can also be ran as just a clusters. [7]. Experiment execution in the IDE is quite customizable.

\subsection{DESIRED FRAMEWORK FOR HOSPITAL MANAGEMENT}

In medical field, preserving affected person data and schedules reasons the era of large quantity of data, in addition this method calls for excessive manpower and time, even though the data are maintained in a desktop. So, introducing the automation device in health facility control reduces time and offers correct reports.

For, this kind of project initially the database has to be created for different module like staff module (along with clinician); patient module with different sub modules involved in their diagnosis and therapy (for providing medical report) and billing module. The test case document will be updated after each process and the test log report will be obtained on completion of the process. 


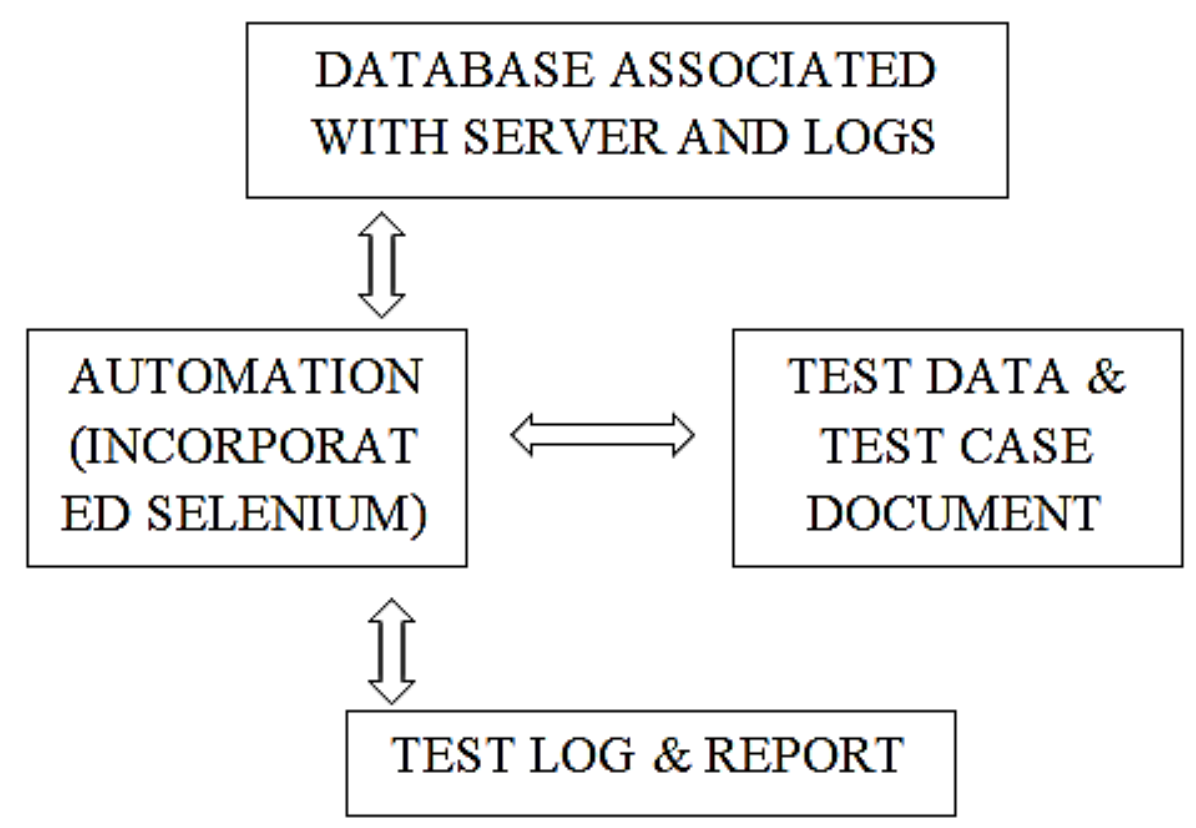

Fig.2: Design of the framework

In staff module the database must be created for the staff and clinician information. The procedure will even contain growing the login call and the password for each login participants. It may be custom designed through integrating the punch in information with the tool. The test case report gets up to date whilst the personnel login. The information up to date will contain the shift information of the staff, the work schedule designed and followed. The test log record may be generated with the up to date information at any time period like hourly, daily, weekly, month-to-month or at any instance. This module isn't complex, so data driven framework [6] is preferred. The framework additionally opts for riding a huge quantity of datasets[10].

The patient module is the maximum vital module to be taken into consideration. The pathway of the patient module will begin with recording the patient info consisting of age, height, weight, blood pressure, $\mathrm{SpO} 2$ and fitness circumstance of the patient. The patient module is labeled into subgroups one for the inpatient and the other for the outpatient. The outpatient can be those patient who've come for short term remedy such as fever, headache etc. (or) people who are under regular checkup. So the outpatient method might also additionally end up with consulting the doctor (or) with minor therapy and diagnosis. The inpatient method may even contain the above processes. The diagnosis and therapy method varies for patients with distinctive health conditions. After diagnosis (scanning and screening) and therapy (intravenous or electric stimulation or laser), the health popularity of the patient could be up to date in the test case report and the very last log document may be obtained. For the inpatient, the other system concerned in the check instances are operation method observed with pre- and post- operative procedures. The system finally ends up with discharge and in this example the log document (i.e. clinical document) is provided to the patient. After discharge if the patient is desired for everyday checkup; then their case report could be 
transferred to the outpatient subgroup. The modularity framework is desired for the patient module because it entails the numerous sub-modules for diagnosis and therapy. The framework will coordinate all of the modules, right into a check log and offer us the document. The billing provided to affected person will contain the length in their admitted period, room provision charges and different remedy charges.

\subsection{EHR vs. SELENIUM}

A digital model of a patient's chart is indeed an electronic health record (EHR). EHRs are true, physician databases which make available information to legitimate users quickly and safely. Whereas an EHR included a detailed medical and therapy histories, it is intended to go beyond normal medical information recorded in a provider's clinic which may include a wider perspective of a patient's condition.

The EHR software has some process limited and it can't be customized according to the requirement of different hospital concern. The processes available are practice management for scheduling and tracking the flow; e-prescribing for managing requests, renewals and for accessing clinical history at any location and timing via any gadget and patient cards for ordering chronologically the patient information. Only the templates are customizable.

Some of the EHR's like NOSH, Solismed, FreeMED and Dolimed are not ONC-ATCB certified. Among them FreeMED and Dolimed have many limitations in their features. In FreeMED the appointment management, e-prescribing and the patient portal maintenance is not possible. In Dolimed e-prescribing and charting features are inconsistent.

The software like MioSalon is supported only in web-based application. In this software, process is good but the log report provided is not in a clarity and detailed manner. The process of staff attendance is done and the values of log report provided is limited; for example the overall timing calculations. The software further requires an alternate browser and multi windows function. The software can't be accessed offline while selenium processes its execution offline. The invoice of the log report for billing purpose can't be edited in case of changing the payment mode, so the process has to be initiated and redone again. The reminder system of the schedule remains ineffective and it seems inconvenient for the users. The one other EHR software, Chirotech also contains many limitation features making the software unproductive. The final log report of the patient statements get complicated, when the process is trialed with mass monthly production. The cloud system of the software remains laggy however fast internet provision is available.

\section{CONCLUSION}

In the below graph, the time duration caused by automation and manual testing is compared for different approaches like smoke, sanity, partial regression and full regression. 


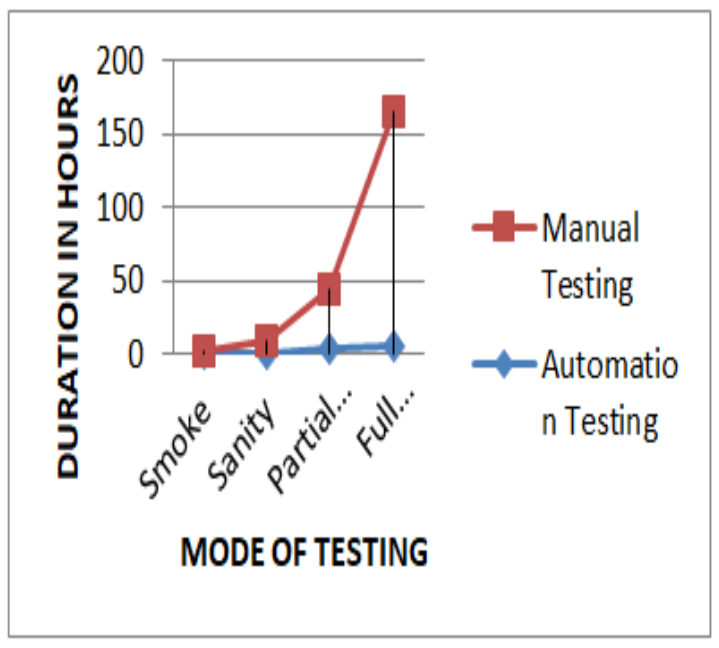

\section{Graph.1: Duration caused by automation testing Vs Manual testing}

The number of test cycles used for analysis of each approaches are as follows, smoke - 10; sanity - 45; partial regression - 200; full regression - 950. The graphical representation reveals that in all the approaches manual testing causes a greater time period while the automation testing acts vice versa.

Many automation tools are available such as Watir, HP-QTP, Selenium, Test Complete, Fitnesse, HP Load Runner, TOSCA, Silktest and TestNG [16]. Among these automation tools, selenium is supported by all major web browsers. The other automation tools than selenium are specific to individual browsers like Internet explorer, Firefox, Google chrome and IF. The languages supported by other automation tools than selenium are also limited and specific which affects the users who may have strength in other programming languages.

Table.1: Comparison of different automation tool

\begin{tabular}{|c|c|c|c|c|c|}
\hline $\begin{array}{c}\text { AUTOMATION } \\
\text { TOOL }\end{array}$ & $\begin{array}{l}\text { OPERATING } \\
\text { SYSTEM }\end{array}$ & $\begin{array}{l}\text { LANGUAGE } \\
\text { S USED }\end{array}$ & $\begin{array}{l}\text { LANGUAGES } \\
\text { SUPPORTED }\end{array}$ & TOOL TYPE & $\begin{array}{c}\text { BROWSER } \\
\text { SUPPORTED }\end{array}$ \\
\hline Selenium & $\begin{array}{l}\text { Cross- } \\
\text { platform }\end{array}$ & Java & $\begin{array}{l}\text { Domain specific } \\
\text { language }\end{array}$ & $\begin{array}{l}\text { Automation tool } \\
\text { for web } \\
\text { applications }\end{array}$ & $\begin{array}{l}\text { Almost all } \\
\text { browsers }\end{array}$ \\
\hline Watir [3] & Cross-platform & Ruby & Java, NET, C\# & $\begin{array}{l}\text { Automation tool } \\
\text { for web } \\
\text { applications }\end{array}$ & Internet explorer \\
\hline HP-QTP & $\begin{array}{l}\text { Microsoft } \\
\text { windows }\end{array}$ & VB script & VB script & $\begin{array}{l}\text { Automation } \\
\text { testing tool }\end{array}$ & $\begin{array}{l}\text { IE, Firefox, } \\
\text { Google chrome }\end{array}$ \\
\hline
\end{tabular}




\begin{tabular}{|c|c|c|c|c|c|}
\hline TOSCA & $\begin{array}{l}\text { Microsoft } \\
\text { windows }\end{array}$ & C\#, java, VB6 & $\begin{array}{l}\text { Java swing, NET, } \\
\text { Dolphi }\end{array}$ & $\begin{array}{l}\text { Automation } \\
\text { testing tool }\end{array}$ & Firefox, IE \\
\hline TestNG & $\begin{array}{l}\text { Windows, } \\
\text { Linac \& MAC }\end{array}$ & Java & Java & $\begin{array}{l}\text { Testing } \\
\text { framework }\end{array}$ & $\begin{array}{l}\text { Firefox, IE, } \\
\text { Chrome }\end{array}$ \\
\hline FitNesse [12] & $\begin{array}{l}\text { Test } \\
\text { Automation } \\
\text { Tool }\end{array}$ & Java & $\begin{array}{l}\text { Dolphi, Ruby, C\#, } \\
\text { C++, Python }\end{array}$ & $\begin{array}{l}\text { Automation } \\
\text { testing tool }\end{array}$ & $\begin{array}{l}\text { Independent of the } \\
\text { browser platforms }\end{array}$ \\
\hline Test Complete & $\begin{array}{l}\text { Test } \\
\text { Automation } \\
\text { Tool }\end{array}$ & Java & $\begin{array}{l}\text { Jscript, VBscript, } \\
\text { Dolphi script, C++ }\end{array}$ & $\begin{array}{l}\text { Automation } \\
\text { testing tool }\end{array}$ & $\begin{array}{l}\text { Firefox, IE, } \\
\text { Chrome }\end{array}$ \\
\hline
\end{tabular}

The above table is a comparison among the important automation tools. The table includes the specification of each automation tool with its parameters such as OS, Languages used and supported, type of the tool and the browser supported.

Selenium is improving at a breakneck speed, with new elements being added on a daily basis. The Selenium designers are extremely responsive to all concerns pertaining to mailing lists for selenium-clients and selenium-level. Selenium is software that automates applications. It's designed for automating web applications for testing purposes, although it's far from limited to that. Exhausting electronic organization errands can be automated as well. A few of the world's largest tech firms have voiced their support for Selenium by integrating it a localized element of its solutions.

This enchant is made as follows while using the Selenium IDE. The html source is handled with Selenium RC so it can be robotized[8]. According the Selenium equipment suite, using Selenium IDE is indeed the simplest and quickest way to create tests (using the capture motor) and play them within the application a short while later. Selenium IDE, a Firefox addon that allows users to easily capture and replay interactions with the application, is beneficial for developing quick bug multiplication scripts and helping with automated processes aided testing. 
So to coordinate the above modules mentioned, the project can either integrate the modularity framework or a hybrid framework, to obtain the effectiveness. Use a hybrid driven framework to prevent complexity when datasets and functionality are both large in number. There are different software tools additionally that play vital position in clinical field. Those software tools encompass EHR software, Medical Database software program, E- prescribing software, Medical billing software, Personal Healthcare software, etc., These software have the negative aspects of inconsistency, inefficiency, chance of being hacked together with terrible in security, will expose the clinical values, that can't be capable of accessing the documents for the duration of low network wherein in selenium, those parameters are highly secured. Selenium has the secured connection and has excessive security. It is consistent and secures the patient's clinical values. Selenium is an open source available and green software program, Framework and language facilities are smooth to enforce the method, time saving, reusable. [2] Execution of the method or assessments is parallel and quicker. Selenium IDE extension is used with Firefox browser, to generate and document for future reference.

\section{REFERENCE}

[1] Monika Sharma et al, "Web based Automation Testing and Tools", (IJCSIT) International Journal of Computer Science and Information Technologies, Vol. 5 (1), 2014, 908-912.

[2] Niranjanamurthy M, Arun Kumar R, Sahana Srinivas, Manoj RK," Research Study on Web ApplicationTesting using Selenium Testing Framework" IJCSMC, Vol 3, Issue 10, October 2014,pg121-126.

[3] I. Singh, B. Tarika, "Comparative Analysis of Open Source Automated Software Testing Tools: Selenium”, International Journal of Information \& Computation Technology. ISSN 0974 -2239 Volume 4, Number 15 (2014), pp. 1507-1518.

[4] Dudekula Mohammad Rafi, Katam Reddy Kiran Moses, K. Petersen and M. V. Mäntylä, "Benefits and limitations of automated software testing: Systematic literature review and practitioner survey," 2012 7th International Workshop on Automation of Software Test (AST), 2012, pp. 36-42, doi: 10.1109/IWAST.2012.6228988.

[5] Monika Sharma, RigzinAngmo, "Selenium Tool: A Web based Automation Testing Framework", International Association of Scientific Innovation and Research (IASIR) (An Association Unifying the Sciences, Engineering, and Applied Research, (2014). ISSN (Print): 2279-0047 ISSN (Online): 2279-0055.

[6] Tuomas Pajumen, Tommi Takala, Mika Katara, "Model-Based Testing a General Purpose Keyword-Driven Test Automation Framework", INTERNATIONAL CONFERENCE ON SOFTWARE TESTING, (2011).

[7] Antawan Holmes, Marc Kellogg, “Automating Functional Test Using Selenium”, AGILE CONFERENCE, (2006).

[8] Chris McMahon, "History of a Large Test Automation Project Using Selenium", AGILE CONFERENCE, (2009).

[9] Andreas Bruns, Andreas Kornstadt, Dennis Wichmann, "Web Application Test With Selenium", IEEE SOFTWARE, (2009).

[10] Martin Fowler, Kent Beck, John Brant, "Refactoring: Improving the Design of Existing Code", U.S., (1999). 
[11] Antawan Holmes, Marc Kellogg, "Automating Functional TestsUsing Selenium", AGILE CONFERENCE, (2006).

[12] Xinchun Wang, Peijie Xu, "Build an Auto Testing Framework Based on Selenium and FitNesse", INTERNATIONAL CONFERENCE ON INFORMATION TECHNOLOGY AND COMPUTER SCIENCE, (2009).

[13] Zhenghua Feng, Ju Gao, Hongwei Zeng, "Research of Web Application Test Automation", COMPUTER ENGINEERING AND DESIGN, (2010).

[14] Harpreet kaur et al Int, "Comparative Study of Automated Testing Tools: Selenium", QUICK TEST PROFESSIONAL AND TESTCOMPLETE. Journal of Engineering Research and Applications, ISSN: 2248-9622, Vol. 3, Issue 5, Sep-Oct 2013, pp.1739-1743.

[15] Deepthi Wilson. R, Manjuprasad. B, 2017, A Comprehensive Review on Selenium Automation Testing Tool, INTERNATIONAL JOURNAL OF ENGINEERING RESEARCH \& TECHNOLOGY (IJERT) NCETEIT - 2017 (Volume 5 - Issue 20).

[16] Hitesh Tahbildar, Bichitra Kalita, "Automated software test data generation: direction of research", INTERNATIONAL JOURNAL OF COMPUTER SCIENCE ENGINEERING SURVEY (IJCSES), (2011). 